\title{
Systematic study for the preparation of Au based catalyst for the glucose oxidation ${ }^{+}$
}

\author{
Eleonora Monti*, Carolina Alejandra Garcia Soto, Francesca Martelli, Alessia Ventimiglia, \\ Nikolaos Dimitratos, Tommaso Tabanelli and Fabrizio Cavani \\ 1 Department of Industrial Chemistry “Toso Montanari”, Alma Mater Studiorum - University of Bologna, \\ Viale del Risorgimento, 4, 40136 Bologna, Italy; carolina.alejandra.garcia.soto@gmail.com (C.G.S.); \\ francesca.martelli10@studio.unibo.it (F.M.); alessia.ventimiglia2@studio.unibo.it (A.V.); \\ nikolaos.dimitratos@unibo.it (N.D.); tommaso.tabanelli@unibo.it (T.T.); fabrizio.cavani@unibo.it (F.C.) \\ * Correspondence: eleonora.monti8@unibo.it; \\ + Presented at the 1st International Electronic Conference on Catalysis Sciences, 10-30 November 2020; \\ Available online: https://sciforum.net/conference/ECCS2020
}

Published: 10 November 2020

\begin{abstract}
Glucaric acid (GA) is one of the top 12 value added chemicals from biomass and this is due to the several applications that this molecule and its derivates can have in the industrial fields. For this reason, it is interesting to find a competitive way to produce it from lignocellulosic feedstock, more in details from glucose. Therefore, our research is focused on the synthesis of GA starting from Glucose in aqueous phase, using molecular oxygen as oxidant agent and gold nanoparticles supported materials as catalysts. All the tests have been carried out in a batch reactor and the catalysts have been prepared using the sol-immobilization method. The role of the stabilizer (polyvinyl alcohol-PVA) has been studied by varying systematically the amount of PVA in the colloidal synthesis and therefore how it could affect (i) the final morphology of the preformed supported metal nanoparticles and (ii) and the catalytic performance in terms of activity, yield and stability; high amount of PVA facilitates the formation of small nanoparticles (no PVA 7.8nm vs PVA2.4, 2.6nm) but also block the active site of the catalyst, giving lower GA yield (22\% vs $17 \%$ ). For this reason, it has been evaluated two different methods to partially remove the PVA, a washing step and several heat treatments, and seems that best results are obtained with the washing and the HT at $120^{\circ} \mathrm{C}$ while at $200^{\circ} \mathrm{C}-250^{\circ} \mathrm{C}$ the average crystallite size increased giving a lower yield of GA.
\end{abstract}

Keywords: glucose; glucaric acid; gold; nanoparticle; sol-immobilization; green chemistry; catalysis; PVA.

Publisher's Note: MDPI stays neutral with regard to jurisdictional claims in published maps and institutional affiliations.

(C) 2020 by the authors. Submitted for possible open access publication under the terms and conditions of the Creative Commons Attribution (CC BY) license (http://creativecommons.org/licenses/by/4.0/). 